Artículo original

\title{
DESARROLLO DE UN ASISTENTE VIRTUAL UTILIZANDO FACEBOOK MESSENGER PARA LA MEJORA DEL SERVICIO DE ATENCIÓN AL CLIENTE EN UNA UNIVERSIDAD PRIVADA
}

\author{
DEVELOPMENT OF A VIRTUAL ASSISTANT USING \\ FACEBOOK MESSENGER FOR THE IMPROVEMENT OF \\ CUSTOMER SERVICE AT THE PRIVATE UNIVERSITY
}

William Condori Quispe ${ }^{1}$

Patrick Cuadros Quiroga ${ }^{1,2}$

Información del artículo:

Recibido: 08/08/2017.

Aceptado: 15/11/2017

${ }^{1}$ Ingeniero de Sistemas

${ }^{2}$ Docente Facultad de Ingeniería Universidad Privada de Tacna

INGENIERÍA

INVESTIGA 
Vol. $1, \mathrm{~N}^{\circ} 1$.

Julio - Diciembre del 2019.

\section{Resumen}

La investigación busca implementar un servicio de atención al cliente en entornos virtuales en la red social Facebook a través de su servicio de comunicación Messenger para brindar un nivel rápido de respuestas y mejorar así el servicio de atención brindado por la Escuela Profesional de Ingeniería de Sistemas de la Universidad Privada de Tacna. Se decidió utilizar un asistente virtual, ya que es un canal en el cual el usuario puede realizar una consulta en modo de conversación haciendo de éste un canal muy amigable. Es así que se desarrolló el asistente virtual en el servicio de mensajería "Messenger" de la Escuela profesional de Ingeniería de Sistemas de la Universidad Privada de Tacna, el cual tiene como función brindar la información solicitada a los respectivos usuarios, atendiendo sus consultas de una manera oportuna y eficaz. Dicha información será gestionada en un administrador de contenidos el cual brindará al usuario una interfaz amigable y sencilla. Se seleccionó "Messenger" como plataforma de mensajería, ya que cuenta con un mayor número de usuarios respecto a los demás servicios, además de contar con controles especiales que hacen posible una mejor interacción entre el asistente y el usuario. El utilizar el asistente virtual trajo consigo una mejora en la calidad de servicio de atención al cliente para la Escuela Profesional de Ingeniería de Sistemas ya que se cuenta con un servicio que está disponible las veinticuatro horas del día y todos los días de año, además de mostrar contenido útil para la comunidad estudiantina de la universidad y para los futuros estudiantes.

PALABRAS CLAVE: Asistente Virtual, Servicio al Cliente, Bot, Facebook Messenger.

\section{Abstract}

The present investigation seeks to implement a customer service in virtual environments in the social network Facebook through its Messenger communication service to provide a rapid level of responses and thus improve the service provided by the Professional School of Engineering Of Systems of the Private University of Tacna. It was decided to use a virtual assistant, since it is a channel in which the user can perform a query in conversation mode making it a very friendly channel. Thus, the virtual assistant was developed in the messenger service "Messenger" of the Professional School of Systems Engineering of the Private University of Tacna, whose function is to provide the requested information to the respective users, attending their queries of a Timely and effective manner. This information will be managed in a content manager which will provide the user with a friendly and simple interface. Messenger was selected as a messaging platform, since it has a greater number of users than other services, in addition to having special controls that make possible a better interaction between the assistant and the user. The use of the virtual assistant brought with it an improvement in the quality of customer service for the Professional School of Systems Engineering since there is a service available 24 hours a day and every day of the year, in addition to Display useful content for the university student community and for prospective students.

KEY WORDS: Virtual Assistant, Costumer Service, Bot, Facebook Messenger.

\section{INTRODUCCIÓN}


El desarrollo de las comunicaciones ha avanzado a pasos agigantados los últimos años, es así que internet y específicamente las redes sociales forman ahora parte de nuestra vida cotidiana, por lo que no es raro observar que se puedan realizar muchas cosas utilizando estas plataformas.

Actualmente el servicio de atención al cliente es una parte fundamental para el éxito de una empresa, Según (Vera, 2007) La Calidad en el servicio son verdadera ventaja competitiva de una organización, el servicio y la atención de calidad son el reflejo del compromiso de quienes integran una institución orientada al cliente, usuario o público en general. Según (Merodio, 2010, pág. 5) "Las redes sociales no son más que la evolución de las tradicionales maneras de comunicación del ser humano....", pero si hablamos de asistentes virtuales, no estamos hablando de un tema nuevo, estos han existido desde antes, pero el llamado "Boom de las redes sociales" y de los servicios de mensajería instantánea han hecho que este término empiece a tomar mucha popularidad, hasta el punto de ser llamado "El futuro de la interacción entre el ser humano y el computador". El Perú no es ajeno a estos cambios.Son ya muchas las empresas que han empezado a optar por el uso de asistentes virtuales o "Bots", ya sea como una alternativa al servicio de atención al cliente o como una alternativa a un asistente de mesa de ayuda (Help Desk). Según (Villar, 2016) empresas como el Banco de Crédito del Perú apostaron por el uso de asistentes virtuales en su página de Facebook. Su nivel de exactitud y su tiempo de respuesta casi inmediata convierten al Bot de RPP en una gran e interesante herramienta que otras empresas optaran por utilizar en un futuro no muy lejano. (Patrón, 2016)

Se ha implementado un asistente virtual cuyo propósito principal es facilitar la búsqueda de información dentro del dominio de la Facultad de Informática de la Universidad Complutense. Dicha funcionalidad está inmersa en un diálogo escrito con el fin de simular el comportamiento de un ser humano y así poder facilitar las búsquedas mejorando la calidad del servicio. (Cubero, 2015). Un Bot conversacional es un software que puede imitar una conversación con una persona utilizando un lenguaje natural. (Valle, López, \& García, 2013) Durán presenta el proceso de implantación del asesor virtual en un portal web, así como también las pruebas de validación realizadas. Presentar así mismo, las recomendaciones para trabajos a realizarse en el futuro dentro de la misma temática. (Durán, 2016). La proliferación de Robots Sociales en la actualidad como sistemas de interacción hombre-máquina, con capacidades cada vez más semejantes a los humanos, está generando una gran variedad de puntos de vista y enfoques en la incorporación de agentes virtuales. (Cobos, 2013)

Un servicio es una actividad o conjunto de actividades de naturaleza casi siempre intangible que se realiza a través de la interacción entre el cliente y el empleado y/o instalaciones físicas de servicio, con el objeto de satisfacerle un deseo o necesidad. (Pérez Torres, 2010) Facebook Messenger es un servicio de mensajería instantánea y aplicación de software. (Zhang, 2011). Actualmente Facebook Messenger se está preparando para llegar a ser una plataforma completa de donde se puedan encontrar aplicaciones juegos, ventas y más gracias a los llamados BOTs. Microsoft Bot Framework es un entorno de trabajo que permite construir y desplegar Bots de alta calidad. Un Bot creado con Bot Framework puede ser desplegado en cualquier servicio accesible, pero es recomendable utilizar Windows Azure (Microsoft, 2017). API.Al es una plataforma de comprensión del lenguaje natural que facilita a que los desarrolladores (y no los desarrolladores) 
diseñen e integren interfaces de usuario conversacionales inteligentes y sofisticadas en aplicaciones móviles, aplicaciones web, dispositivos. BOTs (Api.Ai, 2017) Machine Learning es una herramienta que permite al agente entender las entradas de los usuarios en lenguaje natural y convertirlas en datos estructurados, extrayendo parámetros relevantes. (Api.Ai, 2017). El Diseño Orientado al Dominio promueve la creación de modelos software muy conectado con la lógica del negocio. (Hohpe \& Woolf, 2003). La arquitectura en capas es una buena forma de representar un diseño orientado al dominio (De la Torre, Zorrilla, Ramos, \& Calvarro, 2011).

\section{OBJETIVOS}

- Demostrar que el asistente virtual en la plataforma Facebook Messenger brinda información oportuna y exacta a los usuarios de la Universidad Privada de Tacna.

- Demostrar que el asistente virtual en la plataforma Facebook Messenger puede lograr la aceptación de los usuarios de la Universidad Privada de Tacna.

- Comprobar que el asistente virtual en la plataforma de Facebook Messenger reduce de manera significativa los tiempos de respuesta a los usuarios de la Universidad Privada de Tacna.

\section{METODOLOGÍA}

La presente investigación es experimental y longitudinal de tipo aplicada, correlacional y descriptiva. El desarrollo del sistema fue realizado bajo la metodología de IBM "Rational Unified Process", por ser una metodología que contempla todo el ciclo de vida del software y por ser la metodología estándar más utilizada para el análisis, diseño, implementación y documentación de sistemas orientados a objetos. (Jacobson, Booch , \& Rumbaugh, 1999) El universo poblacional estuvo conformado por los alumnos de la escuela profesional de ingeniería de sistemas de la Universidad Privada de Tacna con una muestra representativa de 52 estudiantes. El desarrollo del asistente virtual para la universidad significa un nuevo canal de comunicación entre la universidad y el estudiante, este nuevo canal debe ser capaz de atender de manera oportuna las consultas realizadas y cumplir con los criterios establecidos para poder así mejorar el servicio de atención al cliente de la universidad. El asistente virtual se encargará de brindar información al usuario de Facebook Messenger, esta información podrá ser vista desde cualquier dispositivo que acepte esta plataforma.

\section{RESULTADOS}

Con respecto a que, si "considera utilizar el asistente virtual cuando necesite información" de la escuela profesional, se puede apreciar que 6 personas (11.8\%) están "Ni de acuerdo, ni en desacuerdo" en considerar utilizar el asistente virtual cuando necesite información de la escuela 
profesional. 24 personas (47.1\%) están "De acuerdo" en que considera utilizar el asistente virtual. 21 personas (41.2\%) están "Totalmente de acuerdo" en que considera utilizar el asistente virtual (fig. 1) 


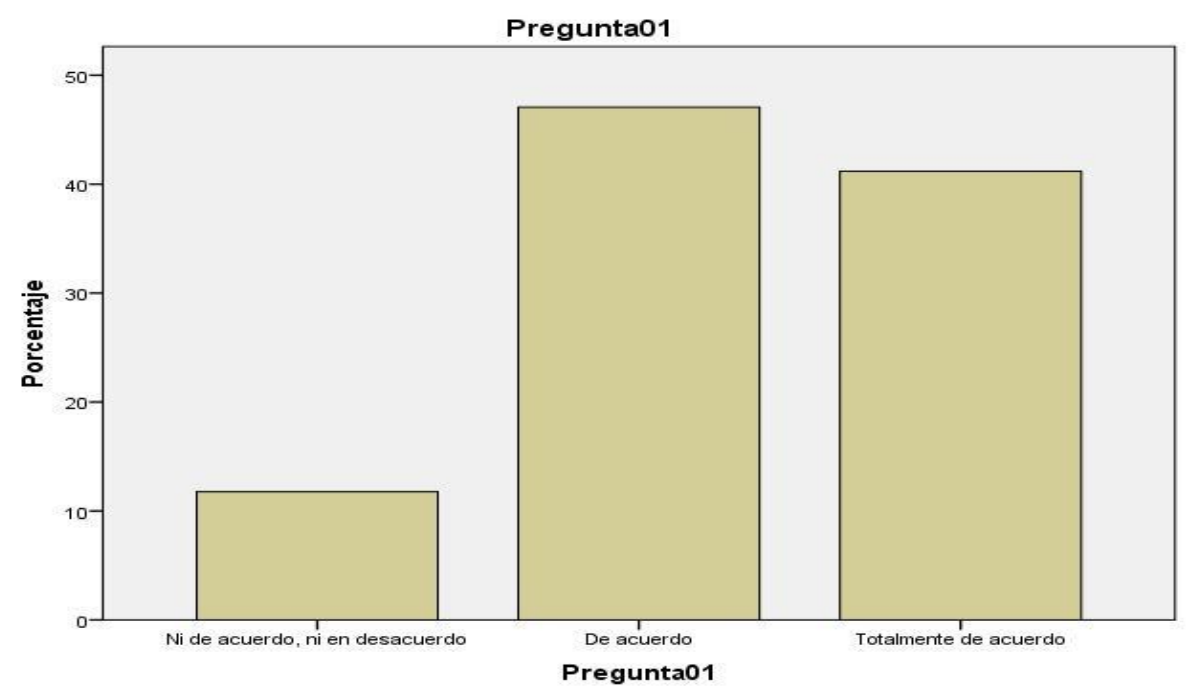

Figura 1: Consideración de uso de asistente virtual”

Según se observa en la tabla 2, con respecto a la oportunidad de entrega de la información. El 1.9\% está "Totalmente en desacuerdo", el 1.9\% está "En desacuerdo", el 38.5\% están "Ni de acuerdo, ni en desacuerdo" y el 55.8\% refiere que la entrega de información es de forma oportuna.

Respecto a que, si el servicio brindado por el asistente virtual está disponible en cualquier momento, el 5.8\% están "Ni de acuerdo, ni en desacuerdo", el $42.3 \%$ están "De acuerdo", el $51.9 \%$ están "Totalmente de acuerdo".

Respecto a que, si las respuestas brindadas por el asistente virtual coinciden con las solicitudes del usuario, el 13.5\% están "En desacuerdo", el 42.3\% están "Ni de acuerdo, ni en desacuerdo" y el $44.2 \%$ están "De acuerdo" en que las respuestas brindadas por el asistente virtual coinciden con las solicitudes del usuario.

Respecto a que, si la forma en que se entrega la información solicitada es el correcto, se puede apreciar que el 9.6\% están "En desacuerdo", el 26.9\% están "Ni de acuerdo, ni en desacuerdo" y el $30.8 \%$ están "De acuerdo".

Respecto a que, si el rendimiento del asistente virtual es el adecuado, se puede apreciar que el 7.7\% están "Totalmente en desacuerdo", el 17.3\% están "En desacuerdo" y el 44.2\% están "Ni de acuerdo, ni en desacuerdo" en que el rendimiento del asistente virtual es el adecuado.

Respecto a que, si el usuario realiza más de una consulta por cada conversación con el asistente virtual, se puede apreciar que el 3.8\% están "Totalmente en desacuerdo", el 13.5\% están "En desacuerdo" y el 30.8\% están “Ni de acuerdo, ni en desacuerdo".

Como se observa en la figura 2, con respecto a que, si considera recomendar el uso del servicio brindado por el asistente virtual, se puede apreciar que el 5.8\% están "Totalmente en desacuerdo", el $13.5 \%$ están "En desacuerdo" y el $48.1 \%$ están "Ni de acuerdo, ni en 
desacuerdo". el 7.7\% están Totalmente de acuerdo en que considera recomendar el uso del servicio brindado por el asistente virtual.

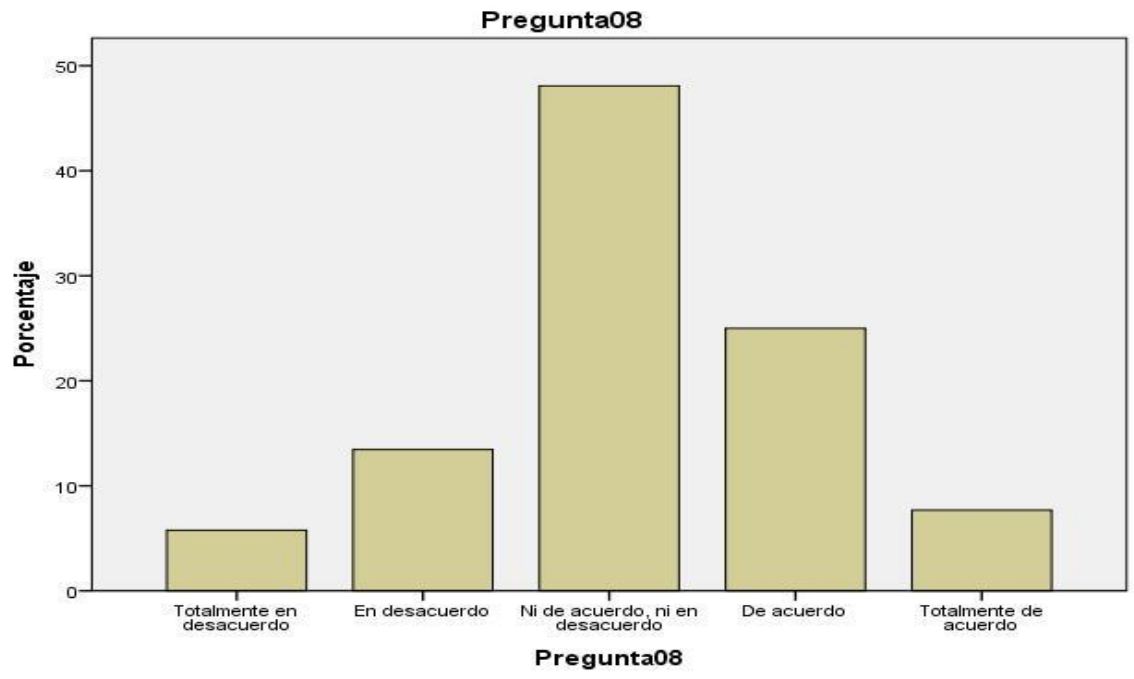

Figura 2: recomendación del asistente virtual

Según se observa en la Fig. 3, con respecto a que, si el usuario utiliza el servicio brindado por el asistente virtual de manera frecuente, se puede apreciar que el $5.8 \%$ están "Totalmente en desacuerdo". el 13.5\% "En desacuerdo", el 34.6\% están "Ni de acuerdo, ni en desacuerdo" y el 46.2\% están "De acuerdo".

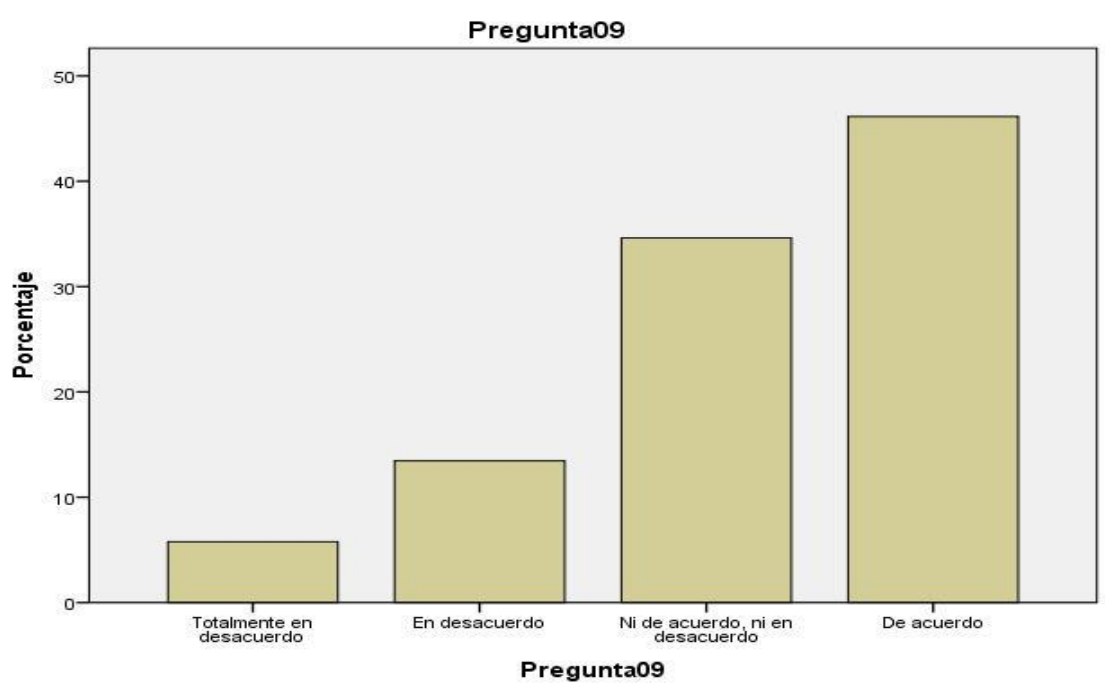

Figura 3: Utilización del asistente virtual

Respecto a que, si considera que la información brindada por el asistente virtual es útil, el $46.2 \%$ están "Ni de acuerdo, ni en desacuerdo", el 53.8\% están "De acuerdo".

Según el indicador de fiabilidad, respecto a que, si la información brindada al usuario solicitante está libre de errores, el 1.9\% está "Totalmente en desacuerdo", el 11.5\% están "En desacuerdo", 
el 21.2\% están "Ni de acuerdo, ni en desacuerdo", el 34.6\% están "De acuerdo" y el 30.8\% están "Totalmente de acuerdo".

Según el indicador de usabilidad, con respecto a que, si es fácil consultar información sobre la escuela profesional de ingeniería de sistemas, el 15.4\% están "Ni de acuerdo, ni en desacuerdo", el 46.2\% están "De acuerdo", y el 38.5\% están "Totalmente de acuerdo".

\section{DISCUSIÓN}

Se concluye que el desarrollo de un asistente virtual para la plataforma Facebook Messenger mejora el servicio de atención al cliente de la Universidad Privada de Tacna. Los resultados muestran las respuestas del usuario las cuales confirman que el desarrollo y posterior implementación de un asistente virtual en la plataforma de Facebook Messenger influye de manera positiva en el servicio de atención al cliente. Se pudo observar la interconectividad del sistema y escalabilidad del sistema obedecen a futuras mejoras del mismo. La portabilidad forma parte de este sistema, una diversidad de plataformas para su uso, desde una PC hasta un Smartphone ya que la plataforma de Facebook Messenger abarca una gran cantidad de dispositivos.

Las respuestas brindadas por el asistente virtual son aun imprecisas en algunos contextos, esto es normal ya que el nivel de precisión aumentara según el tiempo de uso y la cantidad de mensajes con las que cuente. El asistente virtual brinda respuestas oportunas y exactas a los usuarios y ha logrado la aceptación de los usuarios de la plataforma Facebook Messenger. Se comprobó que redujo de manera significativa el tiempo de respuesta a los usuarios que utilizaron el servicio. Se recomienda que el asistente virtual pueda comunicarse con los sistemas existentes, usar los servicios web internos o APIs para lograr una completa integración entre los sistemas, así como incentivar la participación activa de los estudiantes para lograr construir una plataforma más robusta que sea capaz de interactuar con los diferentes sistemas existentes.

\section{REFERENCIAS BIBLIOGRÁFICAS}

Limón, M. A. (2016). Construcción de un prototipo de programa personalizado de tipo chatbot en ambiente java con un lenguaje natural.

Escale. (2015). What is an api?: your guide to the internet business (r)evolution. 3scale.

Abu Shawar, B., \& Atwell, E. (2007). Chatbots: are they really useful? .

Abu, B., \& Atwell, E. (2007). Chatbots: are they really useful? Idv-forum.

Aceves López, J. N. (2013). Importancia de la calidad del servicio al cliente para el funcionamiento de las empresas.

Aguilar, J. E., \& Vargas, J. E. (2010). Servicio al cliente. asociación oaxaqueña de psicología.

Álvarez, R. $(12$ de 04 de 2016). Xataka. obtenido de xataka: https://www.xataka.com/aplicaciones/messenger-platform-asia-es-como-facebookentra-al-mundo-de-los-bots

Api.Ai. (2017). Api docs. obtenido de api docs: https://docs.api.ai/ 
Björnhed, J. (2009). Using a chatbot to prevent identity fraud by social engineering. University of Skövde.

Cobos, J. C. (2013). Integración de un chatbot como habilidad de un robot social con gestor de diálogos.

Cruz, I. (2013). Importancia de la calidad del servicio al cliente. el buzón de pacioli.

Cubero, L. E. (2015). Asistente virtual (chatbot) para la web de la facultad de informática. universidad complutense de Madrid.

David, B., \& Shwartz, S. (2014). Understanding machine learning: from theory of algorithms. cambridge university press.

De La Torre, C., Zorrilla, U., Ramos, M. A., \& Calvarro, J. (2011). Guía de arquitectura n-capas orientada al dominio con .net 4.0. Krasis consulting s.l.

Durán, a. d. (2016). diseño e implantación de un asesor virtual con interfaz web basado en un sistema de gestión de conocimientos y autoaprendizaje. universidad de las fuerzas armadas espe.

fuentes, m., \& gonzález, m. (2013). hacia la interacción en lenguaje natural. Universitat Politécnica de Catalunya.

hohpe, g., \& Woolf, b. (2003). Domain-driven design: tackling complexity in the heart of software. Jacobson, I., Booch , g., \& Rumbaugh, J. (1999). the unified software development process.

Merodio, J. (2010). Marketing en redes sociales. hosteltur.

Microsoft. (2017). Bot framework.com. obtenido de bot framework.com: https://docs.botframework.com

Morris, E., Ancajima, A., Chiri, C., Galindo, J., Guido, C., \& Mejía, E. (2009).

Servicios de contact center basados en offshore outsourcing. esan.

Netcore solutions. (2013). Webhooks integration guide . falconide.

Nilsson, N. (2005). Introduction to machine learning. stanford university.

Patrón, G. (27 de septiembre de 2016). rpp.pe. obtenido de rpp.pe: http://rpp.pe

Pérez Torres, V. C. (2010). calidad total en la atención al cliente.

Polatidis, N. (2012). Chatbot for admissions . university of birmingham.

Rahman, J. (2015). Implementation of alice chatbot as domain specific knowledge bot for brac u (faq bot).

Simonsom, M., \& Thompson, A. (1997). Educational computing foundation (3 ed.). new jersey: prentice-hall.

Spiegel, M., \& Stephens, I. (2009). Estadística. 4ta edición. Mc Graw-Hill.

Tschohl, j. (2008). Servicio al cliente. best sellers .

Valle, L., López, j., \& García, M. (2013). Desarrollo e implementación de un bot conversacional como apoyo a los estudiantes en su proceso de titulación.

Vera, W. (2007). Servicio y atención al cliente. servicios de justicia.

Villar, P. (09 de noviembre de 2016). el comercio. obtenido de el comercio: http://elcomercio.pe Washinton, m. (2016). an introduction to the microsoft bot framework. the ai help.

Zhang, L. (2011). a faster way to message on mobile. 